\title{
Individual level heat risk evaluation using GPS towards smart navigation system
}

\author{
Takahiro Yoshida $^{a, *}$, Yoshiki Yamagata ${ }^{a}$, Daisuke Murakami ${ }^{a}$, b \\ ${ }^{a}$ National Institute for Environmental Studies, Japan, yoshida.takahiro@nies.go.jp, yamagata@nies.go.jp \\ ${ }^{b}$ The Institute of Statistical Mathematics, Japan, dmuraka@ism.ac.jp \\ * Corresponding Author
}

\begin{abstract}
Urban heat risk management is an urgent issue in Japan. Although it is difficult to evaluate heat risk at spatially fine scale due to measurement data restrictions, heat risk varies depending on the location of the people. As a first step of developing smart navigation system for managing heat risk, we try to evaluate personal heat risk by combining ground surface temperature observed from an aircraft and people location acquired from mobile phones at the same time. G star statistic is used to detect high risk areas considering instantaneous co-occurrence of high temperature distribution and walking people density.
\end{abstract}

Keywords: Heat Risk, Exposure, Hazard, Vulnerability, G star statistic, Global Positioning System

\section{Introduction}

Due to urban heat island effect and global warming, health damages caused by heat waves are rising in the world as well as in Japan. In fact, heat strokes caused by large-scale heat waves have been reported in recent years (Rooney et al., 1998; Beniston, 2004; Le Tertre et al., 2006; Hajat et al., 2006; Clark et al., 2010; Steffen, 2014; Richardson, 2015; Klinenberg, 2015; Lemonsu et al., 2015; Mora et al., 2017; Zampieri et al., 2017; Sailor et al., 2019). Improving urban resilience against heat risk is an urgent task (Solecki et al., 2005; Gill et al., 2007; Milan et al.,2015). It is especially important to take measures against heat risks during the summer Olympic Games in 2020 Tokyo.

The heat waves impacts on human health, such as dehydration and dysfunction, become serious when people are exposed to heat for a long time (Rooney et al., 1998; Richardson, 2015). So, to evaluate the heat stress of individual people, it is necessary to know not only heat intensity (i: hazard) but also how people were exposed to the heat (ii: exposure). As temperature changes greatly by location due to the surrounding surface conditions and each individual walking patters are different, it is not easy to evaluate both (i) heat hazard and (ii) exposure of each individual at the same time with conventional methods.

Regarding (i) hazard, the ground surface temperature data is useful to know heat environment in each place. In fact, ground surface temperatures reflect heating on concrete and cooling on parks/grassland more sensitively than air temperatures (Richardson, 2015). The ground surface temperature data observed by the satellite, such as ASTER (Advanced Spaceborne Thermal Emission and Reflection radiometer; Spatial Resolution: 90m) and MODIS (MODerate resolution Imaging Spectroradiometer; Spatial Resolution: $1 \mathrm{~km}$ ), are publicly available in these days. These data would be valuable to analyze thermal condition in a local spatial scale.

Regarding (ii) exposure, collecting and managing location information of people has been done through mobile sensing in recent years. For example, Agoop Co., Ltd. (https : //www . agoop.co.jp/) provides location information logs obtained from applications of smartphones. The logs are acquired by every 30 minutes intervals or $500 \mathrm{~m}$ to $1 \mathrm{~km}$ moves. This Global Positioning System (GPS)'s information will be useful to know movement of individual people, which determines exposure to heat. To find an area in which people are highly exposed to heat hazard, it is necessary to consider spatial cluster of people flows.

Given these backgrounds, as the first step of developing smart navigation system to reduce heat risk of individual people, this study attempts to quantify heat stress of each people by employing ground surface temperature data and mobile sensing data. Furthermore, spatial hots spots of people's heat stress is detected using an approach, which we will develop later, based on the $\mathrm{G}$ star $\left(G^{*}\right)$ statistic (Getis and Ord, 1992), which is a cluster detection approach, which is well-known in spatial statistics (Fischer and Getis, 2009).

The reminder of this study is as follows. Section 2 introduces $G^{*}$ statistic and discusses how to apply this statistic to people flow data. In Section 3, we apply $G^{*}$ statistic to the analysis of heat stress. Finally, in Section 4 we show the future direction of research.

\section{Methodology}

\subsection{G* Statistic}

The $G^{*}$ statistic is a popular test statistic to detect hot spot, such as high-risk area, economically agglomerated area, and ecological community, on a geographical space (Getis and Ord, 1992). The $G^{*}$ statistic for a sample site $i \in$ $\{1, \ldots, n\}$ is defined as follows:

$$
G_{i}^{*}=\frac{\sum_{j=1}^{n} w_{i, j} y_{j}}{\sum_{j=1}^{n} y_{j}},
$$


where where $y_{j}$ represents the observation at $j$-th site. $w_{i, j}$ represents the spatial proximity between the sample sites $i$ and $j$. The proximity can be defined by a variable indicating 1 if the distance between the sites $i$ and $j$ is shorter than a threshold distance, and 0 otherwise. Alternatively, the proximity may be given by a distance-decay function.

The $G_{i}^{*}$ takes a non-negative value. A large $G_{i}^{*}$ suggests a concentration of large $y_{j}$ values nearby the site $j$. For example, if $y_{j}$ represents ground temperatures at the site $j$, the large $G_{i}^{*}$ value means a considerably high ground temperatures nearby that site. The statistical significance can be evaluated analytically, or numerically e.g. using a bootstrap approach. See Getis and Ord (1992) for further details.

Unfortunately, the original $G_{i}^{*}$ statistic assumes samples distributed over a 2-dimensional space. In other words, the statistic is not readily applicable to our GPS data (Figure 1). The next section explains how to apply it to our data.

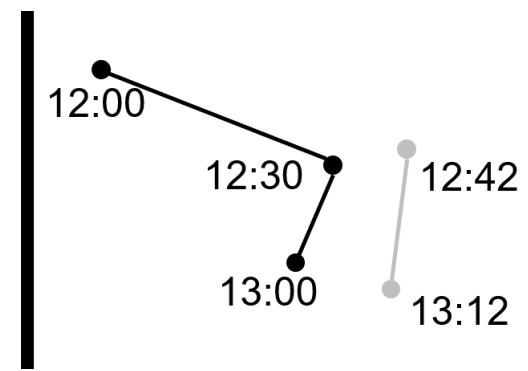

Figure 1. Image of our people flow data

To apply the $G^{*}$ statistic to our data, we need to define the proximity $W_{I, J}$ between the flow $I$ and $J$. The resent spatial statistical papers such as Tao and Thill (2016) and Ermagun and Levinson (2018) have studied proximity between people flows. Based on their discussions, we defined the proximity $W_{I, J}$ as follow:

$$
W_{I, J}=\frac{1}{n_{I} n_{J}} \sum_{i \in I} \sum_{j \in J} w_{I, J} .
$$

In Equation (2), $w_{i, j}$ denotes the spatial proximity between a GPS point $i$, which is in $I$-th flow, and a point, which is in $J$-th flow. We defined the proximity using an exponential decay function $\exp \left(-d_{i, j}\right)$, where $d_{i, j}$ is the Euclidean distance separating these points. Equation (2) evaluates the proximity between flows $I$ and $J$ by averaging the proximities between GPS points in the $I$-th flow and those in the $J$-th flow (see Figure 2).

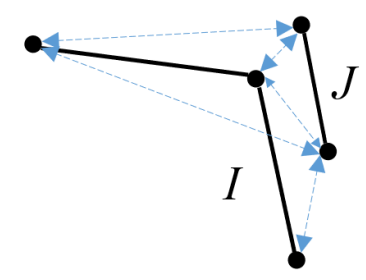

Figure 2. Image of the proximity between people flow $I$ and $J$
Spatial concentration of large $y_{I}$ values can be quantified using Equation (3), which is given by substituting Equation (2) into Equation (1):

$$
G_{I}^{*}=\frac{\sum_{J=1}^{N} W_{I, J} y_{J}}{\sum_{J=1}^{n} y_{J}} .
$$

In our analysis, the $G^{*}$ statistic for flow data (Equation (3)) is used to detect hot spots of people's heat stress. The heat stress of $J$-th people is defined as

$$
y_{J}=\sum_{j \in J} t_{j} \delta\left[g_{j}>30\right]\left(g_{j}-30\right),
$$

where $g_{j}$ is the ground temperature at the $j$-th GPS point, and $t_{j}$ is the estimated time spent at the point, which we assume 0.5 (= 30 minutes),$\delta[\bullet]$ is an indicator returning 1 if

- is satisfied, and 0 otherwise. $y_{J}$ quantifies how long $J$-th people walk on hazardous heat areas, which are defined by areas with the mean ground temperatures being more than 30 degrees at the target period. Equation (3) indicates a large value in areas with many people being suffered from hazardous heats. That is, Equation (3) detects high risk heat areas considering individual people's behavior. Because Equation (3) is identical to the standard $G^{*}$ statistic, the statistical inference for the original $G^{*}$ statistic is read$22_{2}$ availablatistic for People Flow Data

The GPS data we will study is a collection of people locations (2D coordinates) by 30 minutes (see Figure 1), or by $500 \mathrm{~m}$ or $1000 \mathrm{~m}$ of movement for moving people. We focus not on individual GPS points but on flow of individual people indexed by $I \in\{1, \ldots, N\}$. I-th flow is composed of a set of $n_{I}$ spatial coordinates, which we will index by $i \in\left\{1, \ldots, n_{I}\right\}$.

\section{Results and Discussion}

\subsection{Approach}

For ground surface temperatures data, we used thermal image observed from a helicopter (spatial resolution: $5 \mathrm{~m}$; observation period: 11:30-13:00, August 5, 2016; weather: sunny), which is shown in Figure 3. For a data of individual people movement, we used Agoop's "Point type floating population data". We analyze individual level heat stress by applying the approach we developed in the previous section. The target area is the center of Tokyo shown in Figure 3.

Regarding exposure, our targets are pedestrians. This is because pedestrians are directly exposed to high temperatures and solar radiations. Unfortunately, because the abovementioned people flow data do not have attributes such as traffic modes, we need to classify the GPS data into walk, and other transportation modes (see Figures 4 and 5). The criterion of walk is that the average moving speed is less than $6 \mathrm{~km} / \mathrm{h}$ in each movement. $6 \mathrm{~km} / \mathrm{h}$ is equivalent to 1.5 times faster than $4 \mathrm{~km} / \mathrm{h}$ which is often assumed as average walking speed. In addition, when the movement before and after movement at $6 \mathrm{~km} / \mathrm{h}$ is less than $6 \mathrm{~km} / \mathrm{h}$, it is divided into two people flows data (Yamagata et al., 2019). In this 


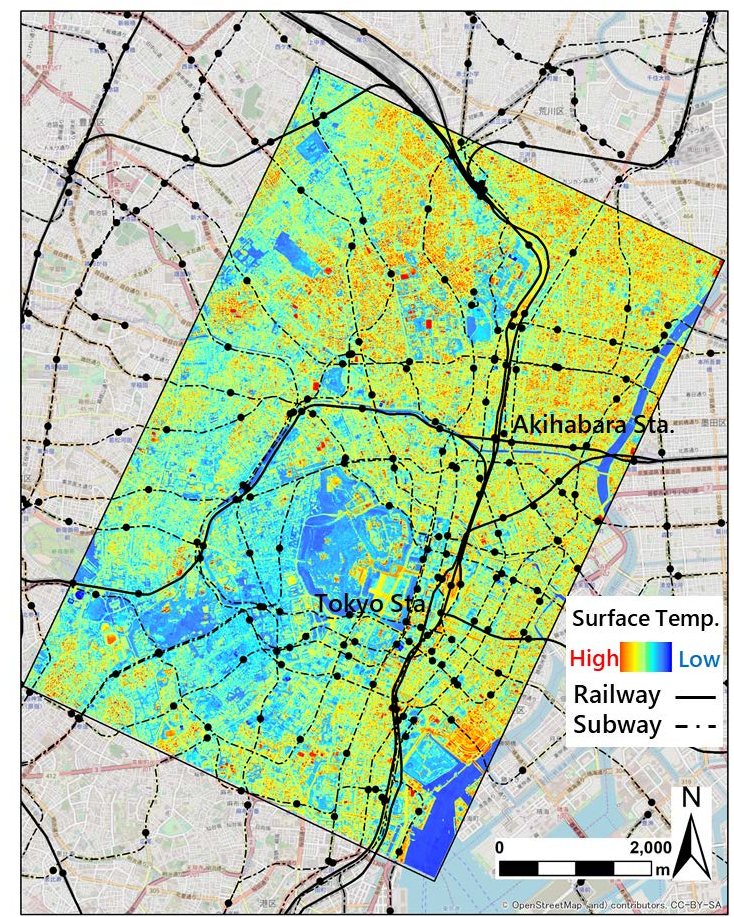

Figure 3. Surface temperature

research, we analyze only people flow tagged by walk extracted by the above assumption. Refinement of the pedestrian identification method is an important subjects in the future.

\subsection{Results}

Figure 6 shows spatial clusters of pedestrians with heat stress evaluated by $G_{I}^{*}$ in Equation (3). $G_{I}^{*}$ is small in areas nearby Tokyo station and Shinbashi station. The result suggests that the heat stress of pedestrians in these two areas is relatively small.

As shown in Figure 3, the ground surface temperature in this two areas is relatively low. Therefore, this result is reasonable. Moreover, it is found that pedestrians with high heat stress are concentrated in near Akihabara Station, Suidobashi Station, Jimbo-cho Station, Hamamatsucho Station. This is probably because the walking distance tends to become longer in these areas where the station density is relatively low. The result suggests that considering the traveling time of pedestrians is important to properly evaluate the heat stress.

\section{Concluding Remarks}

This study developed a new approach to detect high heat risk areas considering people exposure to heat stress using people location information observed by GPS. Then, the proposed approach is tested for the city center area of Tokyo. The result suggests that locational heat stress of individual people changes street by street level. This kind of GPS-based heat risk evaluation approach will be an important step to use geo-sensors towards personal navigation system which could be a very important tool for managing heat risk as well as an adaptation measure to climate change.

We have to address many issues before actually test this system operationally. Firstly, we need to improve the

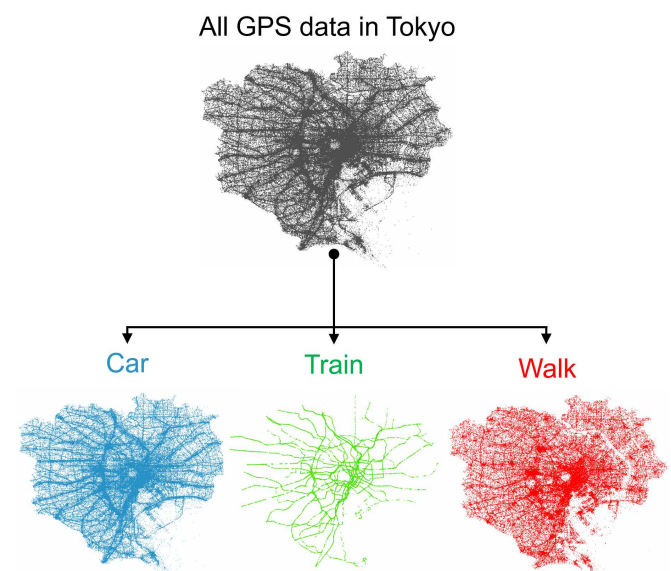

Figure 4. GPS data Classification results

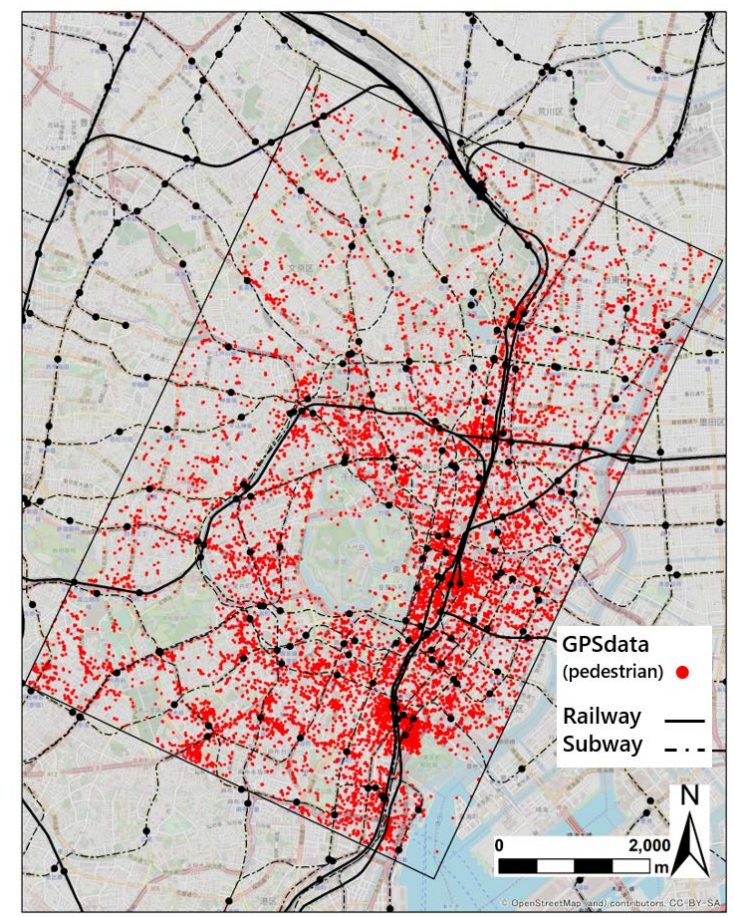

Figure 5. GPS data points of estimated walking pedestrians 


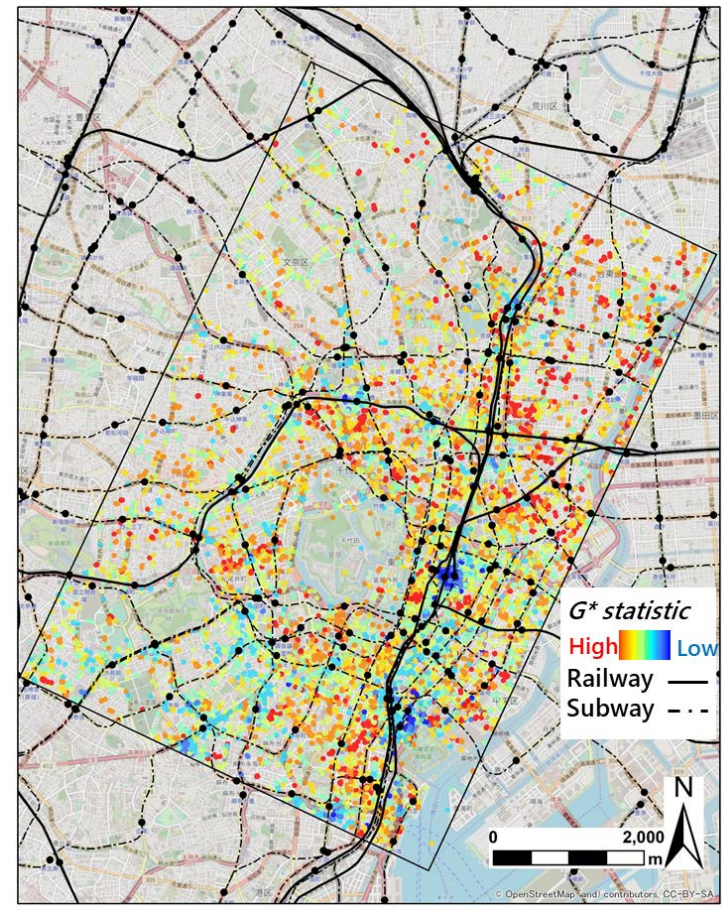

Figure 6. Evaluated $G^{*}$ statistic

pedestrian detection accuracy. For that purpose, supervised machine learning techniques to classify actual pedestrian counts using new GPS sensors will be useful to increase the accuracy. Secondly, we need to improve the data on vulnerability of each individual people. Elders are likely to be more vulnerable to heats than young people (Gonzalez et al., 2008; Worfork, 2000). In fact, combination of improved GPS and socioeconomic data such as age distribution and income are anticipated in the near future. In fact, the use of quasi-zenith satellites is expected to improve the precision of GPS information; the location error is expected to be at most $1 \mathrm{~m}$ for mobile user (Inaba et al., 2009; Hsu et al., 2016; Guo et al., 2017). Thirdly, as heat conditions change dynamically over space and time. A spatiotemporal modeling is needed to predict heat and people behaviors by using multi-scale observations and people sentiments data such as tweets (Yamagata et al., 2015; Murakami et al., 2016).

\section{Acknowledgment}

We appreciate Tokyo Metropolitan Research Institute for Environmental Protection, IDEA consultants, Inc., and Skymap, Inc. for their cooperation on surface temperature measurements. This work was supported by JSPS KAKENHI Grant Number 17H01705.

\section{References}

Beniston, M (2004) The 2003 heat wave in Europe: A shape of things to come? An analysis based on Swiss climatological data and model simulations. Geophys. Res. Lett., 31(2), L02202.

Clark RT, Murphy JM, and Brown SJ (2010) Do global warming targets limit heatwave risk? Geophys. Res. Lett., 37(17), L17703.

Ermagun A and Levinson D (2018) An introduction to the network weight matrix. Geogr. Anal., 50, 76-96.
Fischer MM and Getis A (Eds.) (2009) Handbook of applied spatial analysis: software tools, methods and applications. Berlin: Springer.

Getis A and Ord JK (1992) The analysis of spatial association by use of distance statistics. Geogr. Anal., 24, 189-206.

Gill SE, Handley JF, Ennos AR, and Pauleit S (2007) Adapting cities for climate change: The role of the green infrastructure. Built Environ., 33(1), 115-133.

Guo F, Li X, Zhang X, and Wang J (2017) Assessment of precise orbit and clock products for Galileo, BeiDou, and QZSS from IGS Multi-GNSS Experiment (MGEX). GPS solutions, 21, 279-290.

Hajat S, Armstrong N, Baccini M, Biggeri A, Bisanti L, Russo A, Paldy A, Menne B, and Kosatsky T (2006) Impact of high temperatures on mortality: Is there an added heat wave effect? Epidemiology, 17(6), 632638.

Hsu LT, Gu Y, and Kamijo S (2016) 3D building model-based pedestrian positioning method using GPS/GLONASS/QZSS and its reliability calculation. GPS solutions, 20, 413-428.

Inaba N, Matsumoto A, Hase H, Kogure S, Sawabe M, and Terada K (2009) Design concept of quasi zenith satellite system. Acta Astronaut., 65, 1068-1075.

Klinenberg E (2015) Heat Wave: A Social Autopsy of Disaster in Chicago. Chicago: Univ. of Chicago Press.

Lemonsu A, Viguie V, Daniel M, and Masson V (2015) Vulnerability to heat waves: Impact of urban expansion scenarios on urban heat island and heat stress in Paris (France). Urban Climate, 14, 586-605.

Le Tertre A, Lefranc A, Eilstein D, Declercq C, Medina S, Blanchard M, Chardon B, Fabre P, Filleul L, Jusot JF, Pascal L, Prouvost H, Cassadou S, and Ledrans M (2006) Impact of the 2003 heatwave on all-cause mortality in 9 French Cities. Epidemiology, 17(1), 75-79.

Milan BF and Creutzig F (2015) Reducing urban heat wave risk in the 21 st century. Current Option Environ. Sustainability, 14, 221-231.

Mora C, Dousset B, Caldwell IR, Powell FE, Geronimo RC, Bielecki CR, Counsell CW, Dietrich BS, Johnston ET, Louis LV, Lucas MP, McKenzie MM, Shea AG, Tseng H, Giambelluca TW, Leon IR, Hawkins E, and Trauernicht C (2017) Global risk of deadly heat. Nature Climate Change, 7, 501506.

Murakami D, Gareth WP, Yamagata Y, and Matsui T (2016) Participatory sensing data tweets for microurban real-time resiliency monitoring and risk management. IEEE Access, 4, 347-372.

Richardson H (2015) New world, bringing news and comment on the un to the uk. UNA-UK, Tech. Rep.

Rooney C, McMichael AJ, Kovats RS, and Coleman MP (1998) Excess mortality in England and Wales, and in greater London, during the 1995 heatwave. J. Epidemiol. Commun. Health, 52(8), 482-486.

Sailor DJ, Baniassadi A, O'Lenick CR, and Wilhelmi OV (2019) The growing threat of heat disasters. Environ. Res. Lett., in press.

Solecki WD, Rosenzweig R, Parshall L, Pope G, Clark M, Cox J, and Wiencke M (2005) Mitigation of the heat island effect in urban New Jersey. Global Environ. Change B: Environ. Hazards, 6(1), 39-49.

Steffen W, Hughes L, and Perkins S (2014) Heatwaves: hotter, longer, more often. Climate Council, Australia. 
Tao R and Thill J (2016) Spatial cluster detection in spatial flow data. Geogr. Anal., 48, 355-372.

Worfolk JB (2000) Heat waves: their impact on the health of elders. Geriatr. Nurs., 21, 70-77.

Yamagata Y, Murakami D, Gareth WP, and Matsui T (2015) A spatiotemporal analysis of participatory sensing data tweets and extreme climate events toward real-time urban risk management. arXiv, 1505.06188.

Yamagata Y, Murakami D, Wu Y, Yang P, Yoshida T, and Binder R (2019) Big-data analysis for carbon emission reduction from cars: Towards walkable green smart community. Energy Proc., 158, 4292-4297.

Zampieri M, Ceglar A, Dentener F, and Toreti A (2017) Wheat yield loss attributable to heat waves, drought and water excess at the global, national and subnational scales. Environ. Res. Lett., 12, 064008. 